\title{
Optimal Design of Piezoelectric Actuators for Shunt Damping Techniques
}

\author{
Marcus NEUBAUER \\ Institute of Dynamics and Vibration Research, Leibniz University Hannover \\ ppelstr. 11, 30167 Hannover, Germany; e-mail: neubauer@ids.uni-hannover.de \\ (received October 10, 2014; accepted November 27, 2014)
}

\begin{abstract}
In vibration control with piezoceramics, a high coupling of the piezoelement with the structure is desired. A high coupling improves the damping performance of passive techniques like shunt damping. The coupling can be influenced by a the material properties of the piezoceramics, but also by the placement within the structure and the size of the transducer. Detailed knowlegde about the vibration behavior of the structure is required for this. This paper presents an in-depth analysis of the optimal shape of piezoelectric elements. General results for one-dimensional, but inhomogeneos strain distribution are provided. These results are applied to the case of a longitudinal transducer and a bending bimorph. It is obtained that for maximum coupling, only a certain fracture of the volume should be made of piezoelectric material...
\end{abstract}

Keywords: piezoelectric transducer, piezoelectric shunt damping, piezoelectric energy harvesting, vibration damping.

\section{Introduction}

Piezoceramics are widely used as actuators and sensors in vibration control applications. Due to their large frequency range of operation, they are especially suited for damping of structural vibrations with acoustic emission. The piezoelectric shunt damping technique has been proven to be a lightweight and flexible alternative to fully active vibration techniques and to classical mechanical counteractive measures like tuned mass dampers or materials with high dissipation (Moheimani, 2003). However, the high cost still limits its usage in many cases.

The maximum damping performance of passive shunt damping techniques like the resonant LRnetwork depends on the so-called generalized coupling coefficient of the system (HAGOOD, VON FLOTOW, 1991). This criterion is a measure how strongly the mechanical system can be influenced by the piezoceramics. Generally, the coupling coefficient is different for the individual vibration modes of the structure, and can be influenced especially by the placement of the piezoelement. The generalized coupling coefficient depends on the material coupling factor, but it is typically not the same value. Actually, it is always smaller than the material coupling (UlitKo, 1977).
In order to maximize the performance of the damping device, the placement and size of the transducer must be optimized. Several publications used the method of maximizing the strain energy within the piezoelement to optimize the position. However, this yields only an approximate result, and in some cases more precise methods are required. This publication presents a calculation of the coupling coefficient for an arbitrary vibration mode of the structure, which results in inhomogeneous, but one-directional strain and stress distribution in the piezoelectric material. These results are utilized to give instructions for the optimal design of the most typical piezoelectric transducer, which are the longitudinal and the bending configuration. It is shown that the maximum coupling even occurs for the case that not the whole transducer is made of piezoelectric material. Further on, additional design changes like partitioned electrodes and their influence upon the coupling are discussed.

\section{Piezoelectric equations and generalized coupling coefficient}

In many practical applications, mechanical stress and strain mainly act in one direction. The linear constitutive piezoelectric equations based on IEEE stan- 
dard 176 (IEEE, 1998) then read for the transverse effect

$$
\left[\begin{array}{c}
S_{1} \\
D_{3}
\end{array}\right]=\left[\begin{array}{ll}
s_{11}^{\mathrm{E}} & d_{31} \\
d_{31} & \varepsilon_{33}^{\mathrm{T}}
\end{array}\right]\left[\begin{array}{l}
T_{1} \\
E_{3}
\end{array}\right]
$$

and for the longitudinal effect

$$
\left[\begin{array}{c}
S_{3} \\
D_{3}
\end{array}\right]=\left[\begin{array}{ll}
s_{33}^{\mathrm{E}} & d_{33} \\
d_{33} & \varepsilon_{33}^{\mathrm{T}}
\end{array}\right]\left[\begin{array}{l}
T_{3} \\
E_{3}
\end{array}\right]
$$

The indices denote the axis directions of mechanical and electrical properties, with the 3 -axis being always axis of polarization. Two cases can be distinguished. In longitudinal effect the mechanical stress acts in the same direction as the polarization, while for transversal effect the mechanical stress is orthogonal to the direction of the polarization and the electrical field. The mechanical strain and stress are termed $S$ and $T$, the dielectric charge displacement $D$ and the electrical field strength $E$. The compliance under the condition of a constant electric field is termed $s_{i i}^{\mathrm{E}}$ and $d_{i i}$ is the piezoelectric charge constant. Further on, $\varepsilon_{33}^{\mathrm{T}}$ denotes the absolute permittivity under constant stress. An important derivative parameter for a piezoceramics is the material coupling coefficient $k_{3 i}$, which is defined as

$$
k_{3 i}^{2}=\frac{d_{3 i}^{2}}{s_{i i}^{\mathrm{E}} \varepsilon_{33}^{\mathrm{T}}} .
$$

This parameter can be obtained by a quasistatic deformation cycle of a piezoelectric element. Therefore, a mechanical stress is applied to the piezoceramics with short-circuited electrodes. Consequently, the piezoceramics is mechanically deformed, and a mechanical strain proportional to the stress is obtained. A homogeneous strain and stress distribution in the whole piezoelectric material is assumed. At maximum deformation, the electrodes are isolated, and the mechanical stress is reduced to zero. This reduces the mechanical strain, but a certain amount remains. This remaining deformation is due to the converted energy, and after closing electrodes the piezoelement is deformed back to its original state. Figure 1 illustrates this cycle.

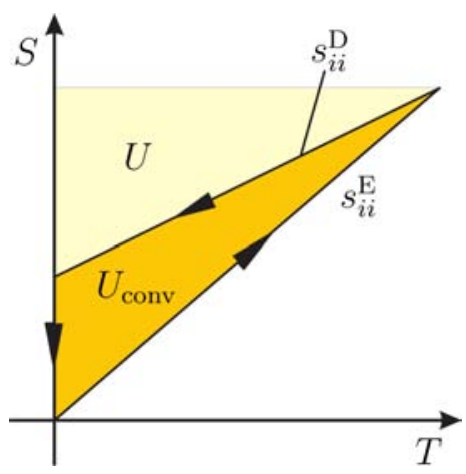

Fig. 1. Quasistatic deformation cycle to determine the material coupling coefficient $k$.
In contrast to this, the generalized coupling coefficient does not only depend on the piezoelement, but also on the overall mechanical structure. It is similarly defined like the mechanical coupling factor, with the potential energy $U$ and the converted energy $U_{\text {conv }}$,

$$
K^{2}=\frac{U_{\mathrm{conv}}}{U+U_{\mathrm{conv}}} .
$$

However, in this case the stress distribution does not have to be homogeneous, and in fact, due to the vibration mode it is typically inhomogeneous. Further on, the potential energy also includes the energy that is stored in the rest of the mechanical system.

A detailed analysis of the potential and the converted energies has been performed in (NEUBAUER, SchWArzendahl, WALlascheK, 2012). A general, but one-dimensional distribution is assumed, which is described by its strain $S_{i}$ in axis direction $i$, its mean strain $\bar{S}_{i}$ and by the variable part $\Delta S_{i, 3}$ over the length of coordinate $x_{3}$,

$$
\begin{aligned}
\bar{S}_{i, 3} & =\frac{\int_{0}^{\ell_{3}} S_{i} \mathrm{~d} x_{3}}{\ell_{3}} \\
\Delta S_{i, 3} & =S_{i}-\bar{S}_{i, 3} .
\end{aligned}
$$

This is illustrated by Fig. 2 .

After some mathematical conversions, the generalized coupling coefficient can be written as

$$
K=\frac{k}{\sqrt{\left(1-k^{2}\right) \frac{\int S_{i}^{2} \mathrm{~d} V}{V \bar{S}_{i}^{2}}+\left(1-k^{2}\right) \frac{\int V}{V S_{i, 3}^{2} \mathrm{~d} V}}+\bar{S}_{i}^{2}} .
$$

This equation showns the relationship between the material coupling and the generalized coupling. Obviously, the generalized coupling $K$ depends beside the material coupling also on the strain distribution within the piezoelectric volume $V$. However, we can conclude that

$$
\frac{\int_{V} S_{i}^{2} d V}{V \bar{S}_{i}^{2}} \geq 1 \quad \text { and } \quad \frac{\int_{V} \Delta S_{i, 3}^{2} \mathrm{~d} V}{V \bar{S}_{i}^{2}} \geq 0
$$

which means that the generalized coupling coefficient is always equal or smaller than the material coupling,

$$
K \leq k_{3 i}
$$

Only for the special case of homogeneous strain distribution these values are equal.

These equations can now be used to study different relevant types of piezoelectric transducers. 


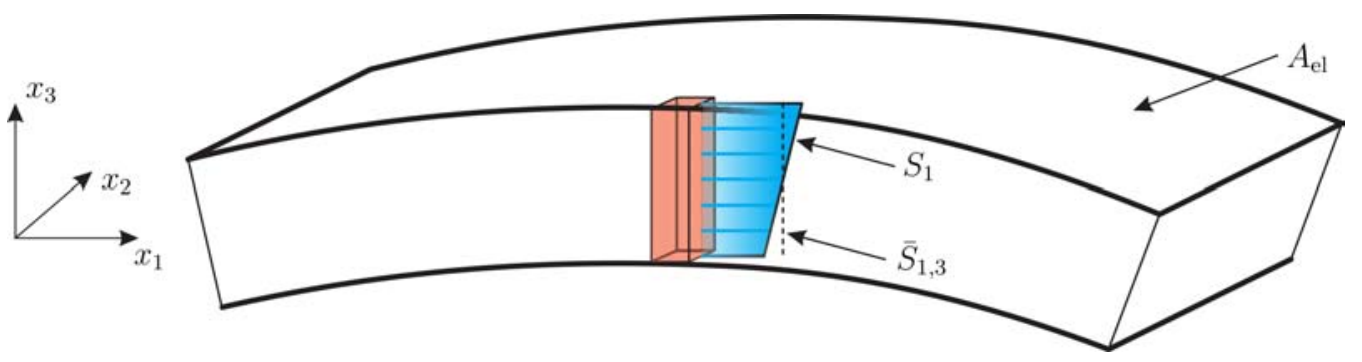

Fig. 2. Piezoelement with one-directional strain distribution.

\section{Longitudinal transducer}

Let us first discuss a longitudinal transducer, Fig. 3. We assume a symmetric transducer with height $L$, of which $\ell_{3}$ is made of piezoelectric material, the rest from passive material. In order to use nondimensional parameters, we substitute these parameters by the ratio $\beta$,

$$
\beta=\frac{\ell_{3}}{L} .
$$

Additionally, we simplify the calculations and assume the Young's moduli of both materials to be equal. In order to obtain the generalized coupling coefficient, we have to determine the strain distribution. We will study two cases: firstly the case of quasistatic deformation Fig. 3a, secondly the first longitudinal vibration mode Fig. 3b.

The quasistatic vibration mode is characterized by a homogeneous strain distribution. Converted and potential energies are straight forward calculated as

$$
\begin{aligned}
U_{\text {conv }} & =\frac{1}{2} \frac{1}{s_{33}^{\mathrm{E}}} \frac{k_{33}^{2}}{1-k_{33}^{2}} \frac{A_{\mathrm{el}}}{\ell_{3}} \beta \hat{q}^{2}, \\
U & =\frac{1}{2} \frac{1}{s_{33}^{\mathrm{E}}} \frac{A_{\mathrm{el}}}{\ell_{3}} \hat{q}^{2},
\end{aligned}
$$

where $\hat{q}$ is the amplitude of deformation. It is understood that the converted energy grows linearly with length ratio $\beta$, while the potential energy is not depending on $\beta$. Substituting into Eq. (4) yields

$$
K=k_{33} \sqrt{\frac{\beta}{k_{33}^{2} \beta+1-k_{33}^{2}}} .
$$

The coupling is not depending on the vibration amplitude $\hat{q}$, but it grows in a nonlinear dependency with $\beta$. Also the material coupling $k$ is included in the result. This is illustrated in Fig. 4. The generalized coupling is normalized to the material coupling, and the dependency with piezovolume $\beta$ is shown for different material couplings $k$. The results differ slightly, but all curves start at zero coupling for $\beta=0$, which is the case without piezoelectric layer. The highest coupling is obtained for $\beta=1$, where the generalized coupling is identical to the material coupling. This result could be anticipated, it is due to the fact that the whole transducer is made of piezoelectric material in this case, and the strain distribution is homogeneous.
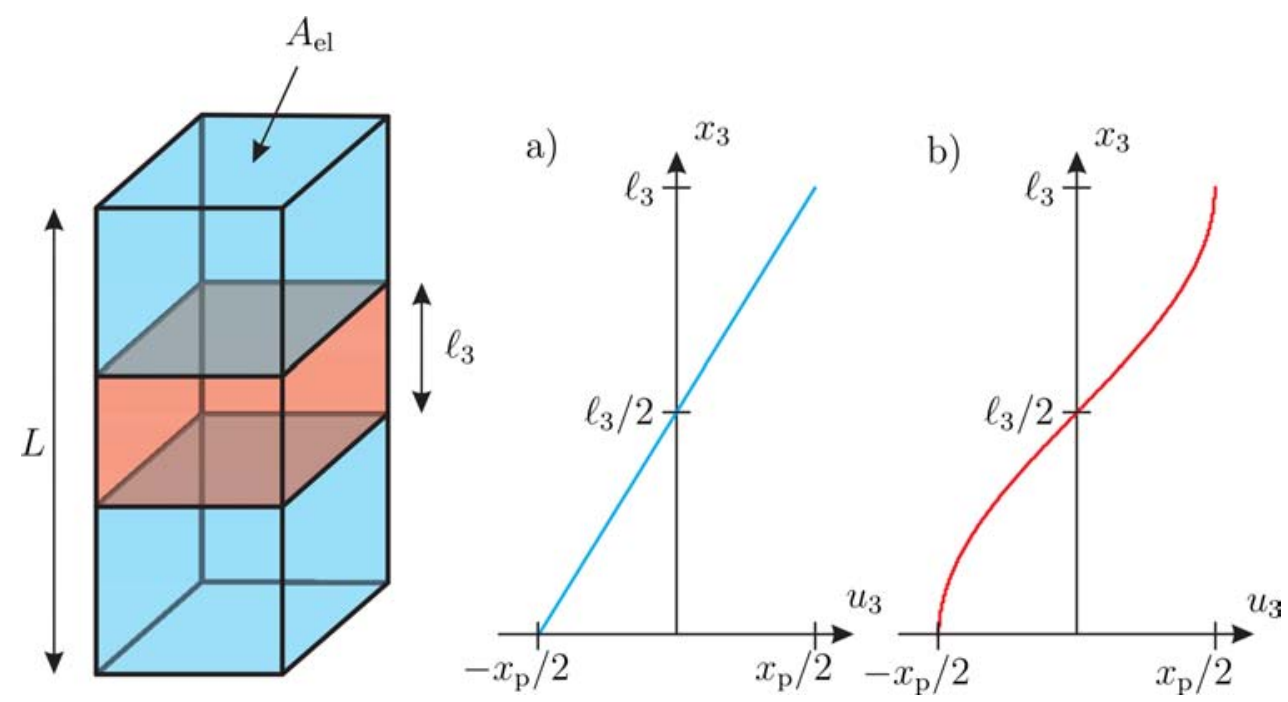

Fig. 3. Longitudinal transducer partially made of piezoelectric material; a) quasistatic deformation, b) first longitudinal vibration mode. 


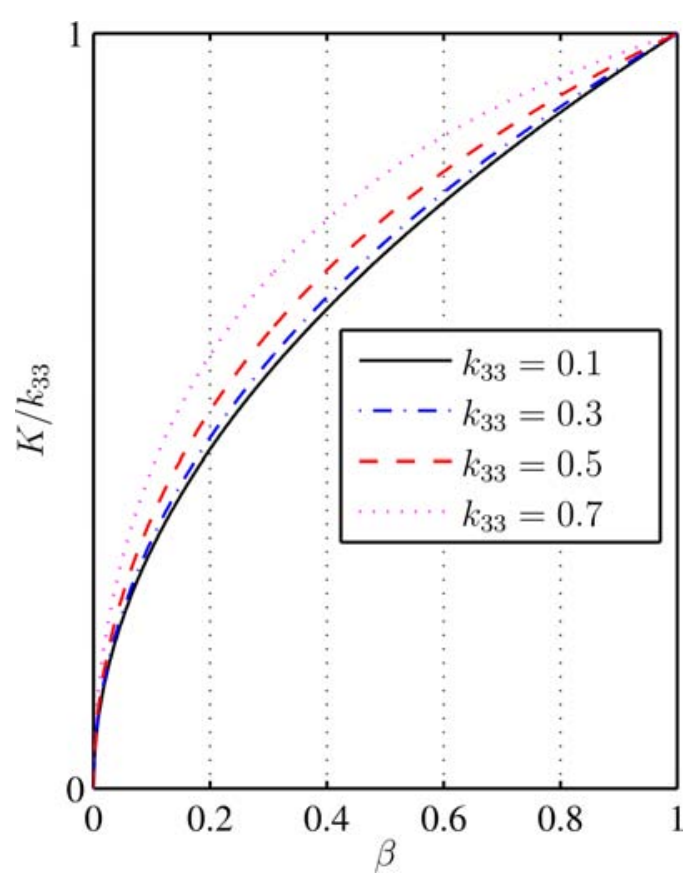

Fig. 4. Generalized coupling factor versus length ratio $\beta$ for different material coupling factors and for quasistatic vibration mode.

A different constellation is obtained when the first longitudinal vibration mode is assumed, with

$$
u_{3}\left(x_{3}\right)=-\frac{1}{2} \cos \left(\frac{\pi}{\ell_{3}} x_{3}\right) \hat{x}_{\mathrm{p}},
$$

which gives a strain distribution

$$
S_{3}=\frac{\partial u_{3}}{\partial x_{3}}=\frac{1}{2} \sin \left(\frac{\pi}{\ell_{3}} x_{3}\right) \frac{\pi}{\ell_{3}} \hat{x}_{\mathrm{p}}
$$

Accordingly, the required mean strain and difference strain values are obtained as

$$
\begin{aligned}
\bar{S}_{i, 3} d & =\frac{1}{\beta L} \int_{\frac{1-\beta}{2} L}^{\frac{1+\beta}{2} L} S_{3} \mathrm{~d} x_{3} \\
& =\frac{\sin \left(\frac{1}{2} \pi \beta\right)}{\beta L} \hat{q}, \\
\Delta S_{i, 3} d & =\frac{1}{2} \frac{\pi}{L} \sin \left(\frac{\pi}{L} x_{3}\right) \hat{q}-\frac{\sin \left(\frac{1}{2} \pi \beta\right)}{\beta L} \hat{q} .
\end{aligned}
$$

It should be noticed that these values only have to be calculated for the piezoelectric volume, see the integration limits. Performing all necessary steps, the generalized coupling coefficient can again be calculated. The result is shown in Fig. 5 in a comparable manner like for the quasistatic deformation. Similar like in the previous case, all functions start at $K=0$ for $\beta=0$ and end at the same value. Inbetween, the characteristics slightly depend on the material coupling $k$.
Secondly we can conclude that even when the whole transducer is made of piezoelectric material, $\beta=1$, the coupling is smaller than the material coupling, strictly speaking it is exactly $\sqrt{8} / \pi=0.9$ of the material coupling. Based on above insights, this reduced coupling is due to the inhomogeneous strain distribution.

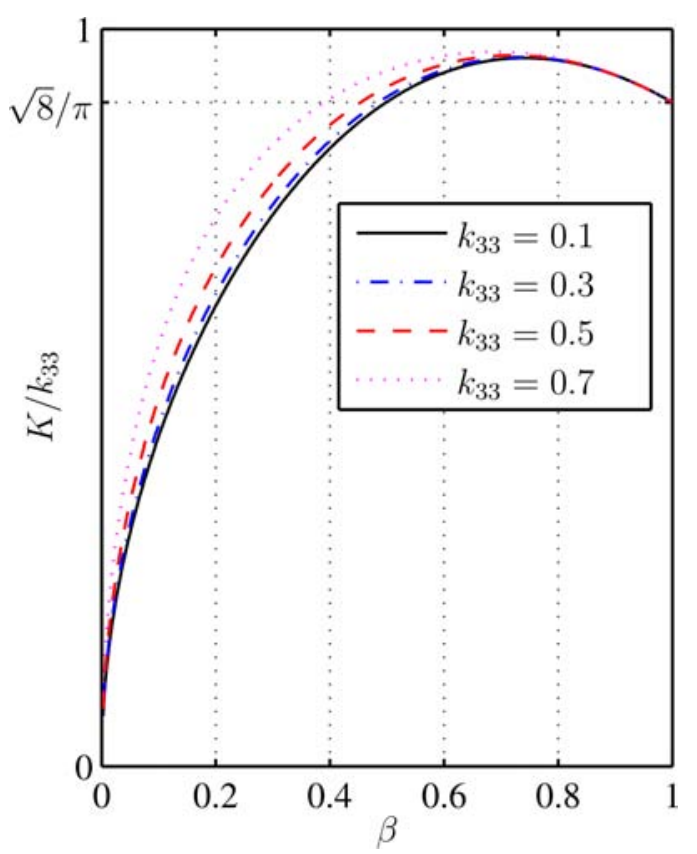

Fig. 5. Generalized coupling factor versus length ratio $\beta$ for different material coupling factors; for first longitudinal vibration mode.

But the most interesting fact is that the generalized coupling coefficient is maximized for a ratio of only about $\beta_{\text {opt }} \approx 0.743$ of the whole transducer. This is against common understanding, which would anticipate the highest coupling for $\beta=1$. However, also this result can be explained by the above findings concerning the inhomogeneous strain distribution. Above that comes the fact that the outer parts of the transducer experience low strain and therefore low energy conversion.

These results say, that more than this optimum $74 \%$ of piezomaterial will actually reduce the generalized coupling from $96 \%$ of $K$ down to $90 \%$ of $K$ for a transducer fully made of piezomaterial. This result is highly important when designing transducer with optimized usage of piezomaterial.

\section{Bending transducer}

The second important type of piezoelectric transducers is the bending type, see Fig. 6. Such bending transducers typically consist of one piezoelectric layer that is placed on a substrate layer. Alternatively, also two piezoelectric layers with opposite poling direction, 


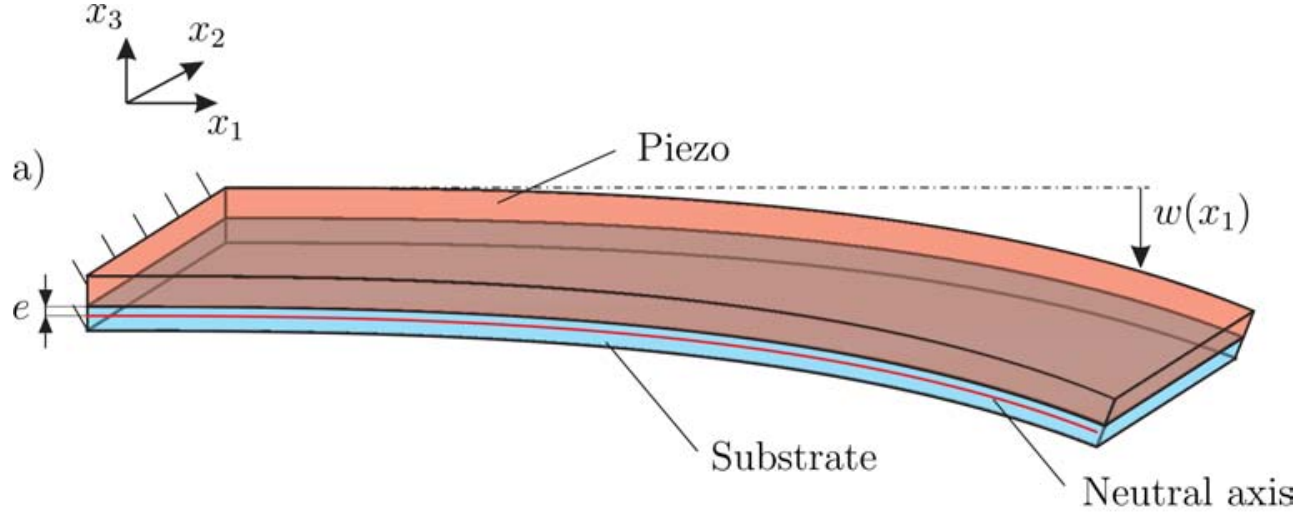

b)

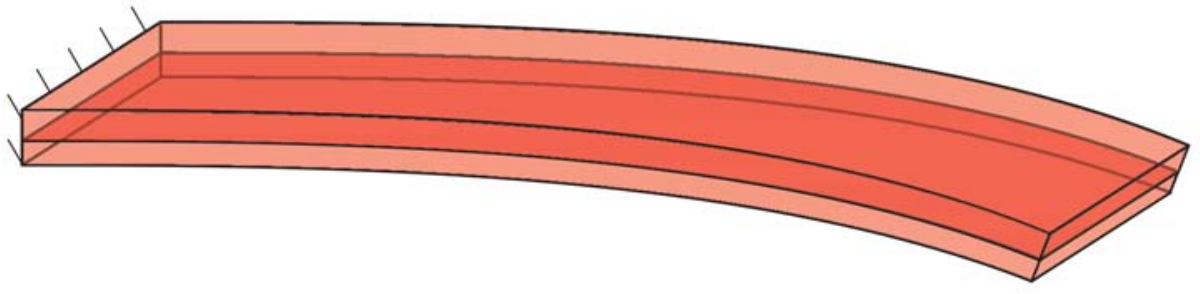

c)

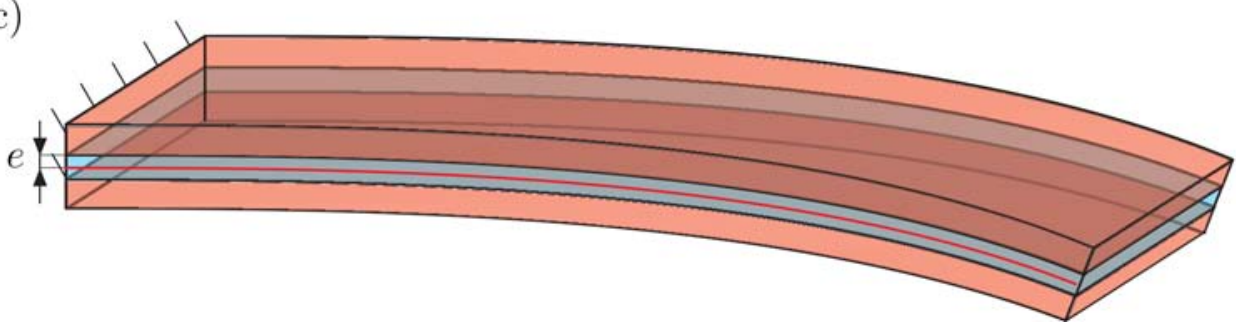

Fig. 6. Different realizations of bending transducers: a) piezoelectric and substrate layers, b) two piezoelectric layers, c) substrate layer between two piezoelectric layers.

or two piezoelectric layers with a substrate layer inbetween can be considered. The same general results for calculation of the generalized coupling factor can be used. The required strain distribution are derived from Euler-Bernoulli assumptions,

$$
S_{1}\left(x_{1}, x_{3}\right)=-\left(x_{3}+e\right) w^{\prime \prime}\left(x_{1}\right) .
$$

In contrast to the longitudinal transducer, this time the transversal effect is utilized, because the mechanical strain acts in $x_{1}$ direction. The parameter $e$ denotes the distance to the neutral axis of the bimorph. It can be noticed that the bending type transducer the strain is never homogeneous. It grows linearly with distance to neutral axis and also to the second derivative of the bending curve $w\left(x_{1}\right)$.

Performing the required mathematical calculations, we end up with the following formula for the generalized coupling coefficient

$$
K^{2}=\frac{k_{31}^{2}}{k_{31}^{2}+\left(1-k_{31}^{2}\right) f F\left[w\left(x_{1}\right)\right]},
$$

see also (WoLF, 2000). All detail informations are summarized in terms $f$ and $F\left[w\left(x_{1}\right)\right]$,

$$
\begin{aligned}
f= & \frac{I_{\mathrm{PZT}}+\frac{1}{r_{\mathrm{E}}} I_{\mathrm{Substr}}^{\mathrm{nF}}}{I_{\mathrm{PZT}}^{\mathrm{nF}}-I_{\mathrm{PZT}}}, \\
F\left[w\left(x_{1}\right)\right]= & \frac{\ell_{1} \int_{0}^{\ell_{1}} w^{\prime \prime 2}\left(x_{1}\right) \mathrm{d} x_{1}}{\left(w^{\prime}\left(\ell_{1}\right)-w^{\prime}(0)\right)^{2}},
\end{aligned}
$$

where $f$ itself depends on the area moments of inertia of the piezolayer and substrate layer. The area moments of inertia with respect to the neutral axis of the whole bimorph are termed $I^{\mathrm{nF}}$, while $I$ denotes the area moment of inertia around the center line of each element. Piezo and substrate layers are defined by the corresponding subindices. The bimorph length is termed $\ell_{1}$, while $w\left(x_{1}\right)$ again determines the bending curvature. The generalized coupling coefficient according to Eq. (16) especially depends on the function $f$ and the functional $F\left[w\left(x_{1}\right)\right]$. While the dependency with the cross-section of the bimorph is included in $f$, the influence of the bending curve $w\left(x_{1}\right)$ and the 
size of electrode can be found in $F\left[w\left(x_{1}\right)\right]$. As the optimization of the cross-section is already performed in (Neubauer, Schwarzendahl, Wallaschek, 2012), these results will just be summarized in short here.

Recalling Eq. (16), the value $f$ must be minimized in order to get the maximum coupling. There are different realizations possible. The most important ones are 1) a piezoelectric layer together with a layer of substrate, 2) two piezoelectric layers, 3) two piezoelectric layers with substrate layer inbetween. We will focus on the third option in the following.

The function $f$ depends on the ratio of Young's moduli $r_{\mathrm{E}}=r_{\mathrm{E} \text {,Piezo }} / r_{\mathrm{E} \text {,Substr }}$ of the piezoelectric and the substrat materials, and also on the area moments of inertia of the substrate and piezoelectric layers. For the symmtric arrangement, these term can be written as

$$
\begin{aligned}
I_{\text {Substr. }}^{\mathrm{nF}} & =\frac{\ell_{2} \ell_{3, \mathrm{~s}}^{3}}{12}, \\
I_{\mathrm{PZT}}^{\mathrm{nF}} & =\frac{\ell_{2} \ell_{3}^{3}}{12}+\ell_{2} \ell_{3}\left(\frac{\ell_{3, \mathrm{~s}}+\ell_{3}}{2}\right)^{2} .
\end{aligned}
$$

They depend on the geometry of the cross section, $\ell_{2}$, $\ell_{3}$ are the corresponding lengths of the piezoelectric layer in direction of $x_{2}, x_{3}$, while $\ell_{3, \mathrm{~s}}$ is the length of the substrate layer (and $\ell_{2, \mathrm{~s}}=\ell_{2}$ ). Figure 7 shows the value $f$ versus the thickness ratio $r_{\mathrm{h}}=\ell_{3} /\left(\ell_{3}+\ell_{3, \mathrm{~s}}\right)$ for different ratios of Young's moduli. For $r_{\mathrm{h}}=0.5$ the transducer only consists of the piezoelectric layer, as the thickness of the substrate layer goes to zero, while for $r_{\mathrm{h}}=0$ the transducer consists of a substrate layer with two piezoelectric layers of vanishing thickness. This graph shows several things:

- for each Young's modulus ratio, there is one optimum thickness ratio which minimizes the function $f$ and therefore maximizes the coupling,

- the smaller the Young's modulus of the substrate, the larger is the optimum thickness of the substrate, and the higher the maximum coupling,

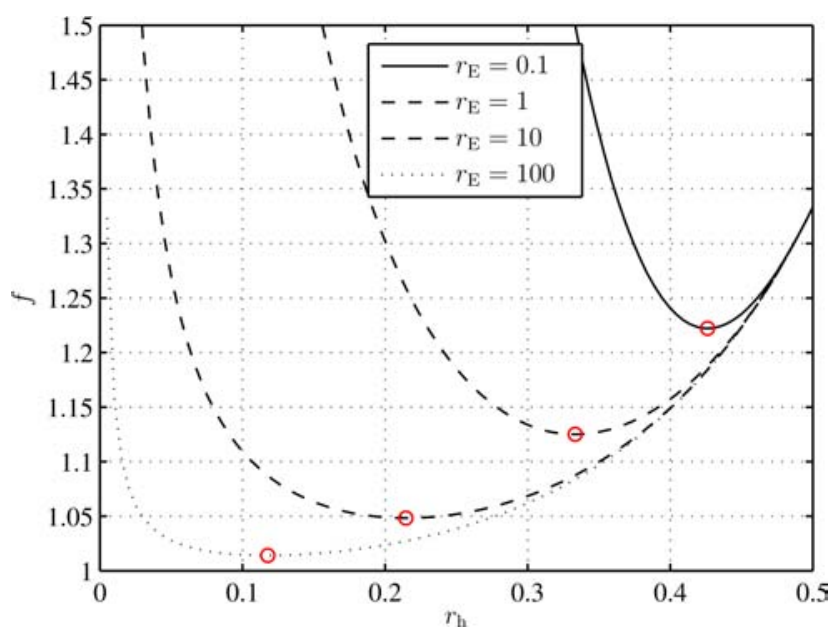

Fig. 7. Functio $f$ versus thickness ratio $r_{\mathrm{h}}$ for various $r_{\mathrm{E}}$.
- for $r_{\mathrm{H}}=0.5$ the coupling is independent from the Young's modulus of the substrate, because it only consists of two piezoelectric layers,

- the generalized coupling of the transducer is always smaller than the material coupling, because the function $f$ is always larger than one.

The optimum cross-sections for different Young's moduli are shown in Fig. 8. The preferable constellation of the cross section is the rightmost one, where the substrate has a (rather theoretical) value of 1000 times the piezoelectric layer.

Similar to $f$, also the functional $F\left[w\left(x_{1}\right)\right]$ must be minimized for high coupling. It can be shown that both $f$ and $F\left[w\left(x_{1}\right)\right]$ can minimally be equal to one, for which the generalized coupling coefficient is again equal to the material coupling.

The results for the coupling depends on the vibration mode, a fact that was already observed for the longitudinal transducer. In this case, the vibration mode is described by the bending curve $w\left(x_{1}\right)$. Again we will analyze the result for two different vibration modes: the quasistatic vibration mode, which would occur for example in the case that a large tip mass is connected to it, and the first bending eigenform which occurs when it is excited in resonance. And also like in the case of the longitudinal transducer, the influence of different sizes of the piezoelectric material is studied. For simplicity, it is assumed that the piezoelectric layer covers the whole length of the bimorph, but the electrode is only from the clamped end until position $d$, see Fig. 6 . The result is given in Fig. 9 versus the ratio $d / \ell_{1}$, which has a similar meaning like the length ratio $\beta$.A similar result can be obtained as for the longitudinal transducer: the coupling of the transducer is increased when the size of the piezoelement resp. the electrode is increased. But again, there exists an optimum length, for which the coupling is maximized (in this case it means that $F\left[w\left(x_{1}\right)\right]$ is minimized). This optimum length is about $2 / 3$ of the beam length for the static case and about $1 / 2$ for the first bending eigenform. The corresponding minimal values for the functional $F\left[w\left(x_{1}\right)\right]$ are 1.14 resp. 1.10, which means the bending transducer does not offer the same amount of coupling as the material factor. The reason for this is again the inhomogeneous strain distribution within the piezoelectric layer. Above that comes a further reduction due to the cross-section, see (Neubauer, Schwarzendahl, Wallaschek, 2011; 2012). These results again prove that it is possible to safe a considerable amount of piezoelectric material while even increasing the generalized coupling of the transducer.

Finally, also the possibility of a partition of the electrodes will be discussed. We study a partition into individual electrodes with equal sizes, but with different numbers of electrodes. For example, in the case of two electrodes the first electrode covers the first half 


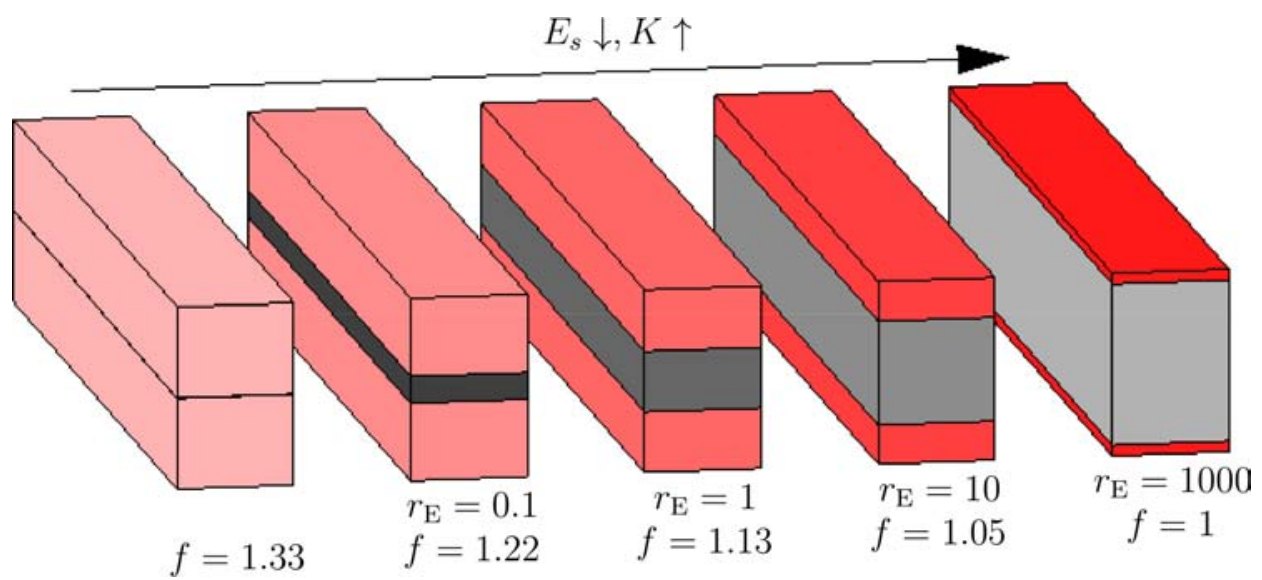

Fig. 8. Optimal cross sections for bending transducers. Maximum coupling grows from left to right.

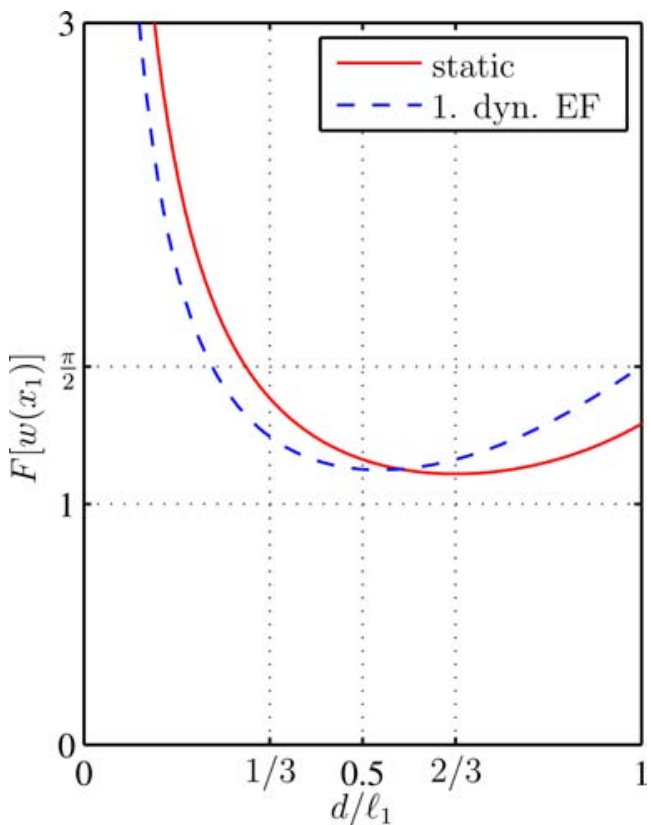

Fig. 9. Functionals $F\left[w\left(x_{1}\right)\right]$ versus length of electrode for static and first bending eigenform.

of the length and the second one the second half of the beam, while for three electrodes they cover the first, the middle and the last third of the length. The corresponding coupling is again analyzed by the values of the functional $F\left[w\left(x_{1}\right)\right]$, which are presented in Fig. 10. This result shows that a fine partition generally increases the coupling, at least in most cases. By checking the values for the static and the first eigenform, the functional approaches quickly the theoretical optimum of $F\left[w\left(x_{1}\right)\right]=1$. A partition into two electrodes on each surface is already sufficient, a further increase does not increase the coupling any significantly further. However, for the higher eigenforms the partition has a strong impact and improves the coupling. This is due to the fact that in these cases the strain

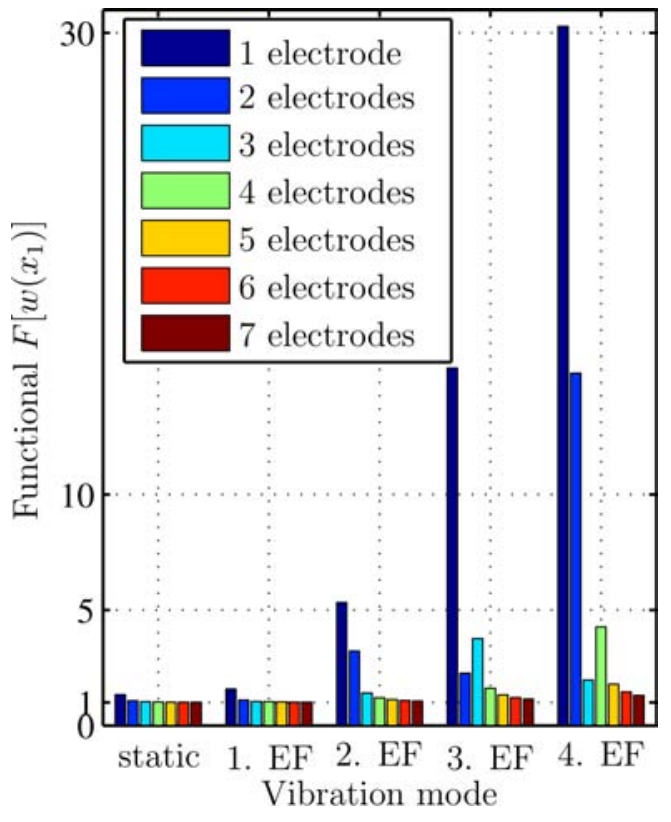

Fig. 10. Functional $F\left[w\left(x_{1}\right)\right]$ for static and first 4 eigenforms or the beam with equally spaced partition of the electrodes. changes its sign over the length of the beam, with alternating areas of tension and compression forces. Using only one electrode would result in an internal short cut of the piezoelement, and the overall coupling is very low, which can be seen on the high values of the functional.

\section{Conclusions}

This paper present an in depth analysis of the generalized coupling coefficient of piezoelectric transducer. The coupling is generally obtained for one-dimensional strain distributions. The results are then applied to longitudinal and bending transducers. They are used to optimize the usage of piezoelectric material. 
It is shown that for inhomogeneous strain distributions the generalized coupling is reduced compared to the material coupling. For the longitudinal transducer, an amount of about $74 \%$ piezoelectric material results in the highest coupling, while the bending transducer should be covered only by $1 / 2$ of its length with piezoelectric material. For the bending transducer, the optimal electrode coverage must be combined with the optimal cross section of substrate and piezoelectric layer.

\section{References}

1. Hagood N.W, von Flotow A. (1991), Damping of structural vibrations with piezoelectric materials and passive electrical networks, Journal of Sound Vibration, 146, 243-268, doi: 10.1016/0022-460X(91)90762-9.

2. IEEE (1889), Ieee standard on piezoelectricity, ANSI/IEEE Std 176-1987.
3. Moheimani S.O.R. (2003), A survey of recent innovations in vibration damping and control using shunted piezoelectric transducer, IEEE Transactions on Control Systems Technology, 11, 4, 482-494.

4. Neubauer M., Schwarzendahl S.M., WaLLASCHEK J. (2011), Determination of the piezoelectric coupling coefficient for arbitrary deformation, Proceedings of Mechatronic Systems and Materials (MSM) Conference, Kaunas.

5. Neubauer M., Schwarzendahl S., WallascheK J. (2012), A new solution for the determination of the generalized coupling coefficient for piezoelectric systems, Journal of Vibroengineering, 14, 1, 105-110.

6. Ulitko A.F. (1977), Theory of electromechanical energy conversion in nonuniformly deformable piezoceramics, Soviet Applied Mechanics, 13, 1055-1062, doi: 10.1007/BF00883190.

7. Wolf K. (2000), Electromechanical Energy Conversion in Asymmetric Piezoelectric Bending Actuators, PhD thesis. 